TRANSACTIONS OF THE

AMERICAN MATHEMATICAL SOCIETY

Volume 349, Number 5, May 1997, Pages 1993-2006

S 0002-9947(97)01876-X

\title{
CLASSIFICATION PROBLEMS FOR SHIFTS ON MODULES OVER A PRINCIPAL IDEAL DOMAIN
}

\author{
FABIO FAGNANI AND SANDRO ZAMPIERI
}

\begin{abstract}
In this paper we study symbolic dynamics over alphabets which are modules over a principal ideal domain, considering topological shifts which are also submodules. Our main result is the classification, up to algebraic and topological conjugacy, of the torsion-free, transitive, finite memory shifts.
\end{abstract}

\section{Introduction AND STATEMENT OF THE RESUlt}

Introduction. Most familiar systems in symbolic dynamics are topological shifts over finite alphabets. Recently, however, there has been some interest in considering also more general infinite alphabets. This type of generalization is a natural one to make and it comes up in various fields such as differentiable dynamics [4], nonuniqueness of equilibrium states in statistical mechanics [11], formal languages and automata $[1,2]$, and also in more applicative contexts, such as coding for magnetic resonances [17]. In the mentioned papers Markovian shifts over countable alphabets are considered and questions like recurrence, topological entropy, ergodic measures are addressed. Further results of this fashion can be found in [18].

On the other hand, there has also been growing interest in considering topological shifts endowed with group structure $[9,10,5,6]$. In this case we usually consider alphabets with a group structure and we then study shifts over such alphabets which are in addition subgroups of the infinite product group: they are called group shifts. The group structure in general gives strong limitations on the type of dynamical properties that the shift can possess, so that such dynamical systems are, in some cases, particularly simple to study. However, the group structure also leads to new questions such as classification from an algebraic-topological point of view and the related problems of finding new invariants. In this context, most of the interest has been until now focused on the case when the alphabet is a finite or compact group, especially for the important role that such systems play in the general theory of automorphisms of compact groups [16, 10, 19]. Compactness permits one to elaborate a quite elegant and unified theory: a striking result is that every group shift over a compact group is of finite type. Non-compact groups have been considered in [6].

Shifts over groups have also recently appeared in coding theory [7], since they seem a promising generalization of convolutional codes over finite fields. On the

Received by the editors November 15, 1995.

1991 Mathematics Subject Classification. Primary 54C70, 15A23, 93B25.

Key words and phrases. Shifts, dynamical systems, transitivity, principal ideal domains, polynomial matrices, behavioral approach. 
other hand, shifts endowed with richer algebraic structure, such as that of a module over a ring, have important connections with control theory [20, 21, 22, 3].

In this paper we study symbolic dynamics over alphabets which are finitely generated modules over a principal ideal domain. In the case when the ring is $\mathbf{Z}$ we are indeed considering group shifts over finitely generated Abelian groups, and so our results are of interest in the general framework of symbolic dynamics over infinite (non-compact) groups. On the other hand, the fact of working with general principal ideal domains does not require much more effort, and permits us to achieve results which are more general and of potential use also in such other fields as systems and control theory: indeed, when the ring is $\mathbf{R}[x]$, our results can be suitably interpreted in the context of linear control systems with a parameter.

$R$-shifts, memory and limit rank. In the sequel $R$ will always denote a principal ideal domain with identity (PID) and all the finitely generated $R$-modules will be assumed to be equipped with the discrete topology. Let $V$ be such an $R$-module and consider the product $R$-module $V^{\mathbf{Z}}$ equipped with the product topology on which the shift $\sigma$ acts: $((\sigma v)(t):=v(t+1))$. Let $\mathcal{S} \subseteq V^{\mathbf{Z}}$ be a closed $\sigma$-invariant $R$-submodule. Then, the dynamical system $\left(\mathcal{S}, \sigma_{\mid \mathcal{S}}\right)$ is called an $R$-shift over the alphabet $V .\left(V^{\mathbf{Z}}, \sigma\right)$ is called the full $R$-shift over $V$. For the sake of simplicity, whenever this does not cause confusion, the restriction sign in $\sigma$ will be dropped. Also, we will refer to $\mathcal{S}$ itself as to the $R$-shift $(\mathcal{S}, \sigma)$.

If $I \subseteq \mathbf{Z}$, denote by $\mathcal{S}_{\mid I}$ the $R$-module of restrictions to $I$ of the bi-infinite sequences in $\mathcal{S}$. $\mathcal{S}$ is said to have memory $n \in \mathbf{N}$ if

$$
v \in V^{\mathbf{Z}} \text { and } v_{[[t, t+n]} \in \mathcal{S}_{[[t, t+n]} \forall t \in \mathbf{Z} \Rightarrow v \in \mathcal{S} .
$$

$\mathcal{S}$ is said to have finite memory (or to be of finite type), if it has memory $n$ for some $n \in \mathbf{N}$. If $V$ is Artinian, every $R$-shift over $V$ automatically has finite memory [22]. Non-finite memory shifts over modules do exist: see [6] for an example with $V=R=\mathbf{Z}$.

Every finitely generated $R$-module $V$ can be decomposed as $V=F \oplus T$ where $T=T(V)$ is the torsion $R$-submodule of $V$ and $F$ is $R$-free. The $R$-rank of $V$ $\left(\mathrm{rk}_{R} V\right)$ is defined as the $R$-dimension of $F$. Let $\mathcal{S} \subseteq V^{\mathbf{Z}}$ be an $R$-shift. Consider the mapping $n \mapsto \operatorname{rk}_{R}\left(\mathcal{S}_{\mid[1, n]}\right)$. We can easily see that it is subadditive. It is then a standard fact that there exists

$$
L(\mathcal{S}):=\lim _{n \rightarrow+\infty} \frac{\operatorname{rk}_{R}\left(\mathcal{S}_{\mid[1, n]}\right)}{n}
$$

which will be called the limit rank of $\mathcal{S}$. Clearly, we always have that $L(\mathcal{S}) \leq \operatorname{rk}_{R} V$. It can be shown ([6], Proposition 3.2) that $L(\mathcal{S})$ is a non-negative integer if $\mathcal{S}$ has finite memory.

$R$-morphisms. Consider two $R$-shifts $\mathcal{S}_{1}$ and $\mathcal{S}_{2}$. A morphism of dynamical systems $\psi: \mathcal{S}_{1} \rightarrow \mathcal{S}_{2}$ (i.e. $\psi$ is continuous and $\psi \circ \sigma=\sigma \circ \psi$ ) is called an $R$-morphism if in addition it is an $R$-homomorphism. If, moreover, $\psi$ is invertible, namely a conjugacy, $\psi$ is called an $R$-conjugacy. In this last case, $\mathcal{S}_{1}$ and $\mathcal{S}_{2}$ are said to be $R$-conjugated, and we write $\mathcal{S}_{1} \simeq_{R} \mathcal{S}_{2}$.

We have the following simple result.

Proposition 1. Let $V, W$ be finitely generated $R$-modules and let $\mathcal{S} \subseteq V^{\mathbf{Z}}$ be an $R$-shift. A map $\phi: \mathcal{S} \rightarrow W^{\mathbf{Z}}$ is an $R$-morphism if and only if there exists numbers 
$h, k \in \mathbf{N}$ and $\zeta \in \operatorname{Hom}_{R}\left(\mathcal{S}_{\mid[0, h+k]}, W\right)$ such that

$$
\phi(v)(t)=\zeta\left(v_{\mid[t-h, t+k]}\right) \quad \forall v \in \mathcal{S}, t \in \mathbf{Z} .
$$

Proof. Straightforward.

$\phi$ in (3) is said to have memory $k$ and anticipation $h$. In the case $h=k=0$, we will also use the notation $\phi=\zeta^{\infty}$.

Remark. It follows from Proposition 1, using the same ideas as in [6] (see Propositions 1.2 and 1.8), that finite memory and limit rank are invariant under $R$ conjugacies.

Main results. The following is one of the main results of this paper.

Theorem 2. Let $\mathcal{S}_{1}$ and $\mathcal{S}_{2}$ be two $R$-shifts which are transitive, finite memory and $R$-torsion free. Then,

$$
\mathcal{S}_{1} \simeq_{R} \mathcal{S}_{2} \quad \Leftrightarrow \quad L\left(\mathcal{S}_{1}\right)=L\left(\mathcal{S}_{2}\right)
$$

In particular, if $\mathcal{S}$ is an $R$-shift which is transitive, finite memory and $R$-torsion free, then

$$
\mathcal{S} \simeq_{R}\left(R^{L(\mathcal{S})}\right)^{\mathbf{Z}}
$$

Remark. In case $R$ is a field, Theorem 2 was essentially proven in [21].

In case torsion is present it is obvious that the limit rank is no longer sufficient to separate $R$-conjugacy classes of transitive, finite memory $R$-shifts. Actually, it is shown in $[9,5]$ that there exist transitive, finite memory $\mathbf{Z}$-shifts which are not $\mathbf{Z}$-conjugated to full $\mathbf{Z}$-shifts. A related simpler question which can be posed in this more general setting is which are the conditions, beside transitivity and finite memory, which characterize the $R$-shifts which are $R$-conjugated to a full $R$-shift. This question has been answered in [5] for $R=\mathbf{Z}$ and $V$ a torsion $R$-module. Theorem 11 generalizes this result to the case when $R$ is any PID and $V$ is still a torsion $R$-module: it provides necessary and sufficient conditions for an $R$-shift $\mathcal{S}$ to be $R$-conjugated to a full $R$-shift in terms of the transitivity of certain $R$-subshifts of $\mathcal{S}$. Corollary 15 is a further generalization of Theorem 11 to the case when $V$ is any finitely generated $R$-module, and is our more general result on this issue. The proof of Corollary 15 is based on Theorem 14, which yields a nice torsion-free splitting for $R$-shifts.

Finally, notice the following implication of Theorem 2: since $R^{l}$ and $R$ are homeomorphic when equipped with the discrete topology, it follows that every $R$-shift $\mathcal{S}$ which is transitive, finite memory and $R$-torsion free, is topologically conjugated to the full shift $R^{\mathbf{Z}}$. Actually, using the techniques of $[9,10]$, it is not difficult to prove that every transitive finite memory $R$-shift is topologically conjugated to a full shift.

Outline of the contents. This paper is structured as follows. In Section 2 we establish certain results for $R$-morphisms between full $R$-shifts over free modules. These results, beside their intrinsic interest, are used to prove Theorem 10 in Section 3 , which immediately yields Theorem 2 in the case when the alphabets of the $R$ shifts are given by free $R$-modules. After that, we pass to consider $R$-shifts over torsion modules and we prove Theorem 11. Finally we pass to consider the case when the alphabet contains both a torsion and a free part. After a series of auxiliary 
results, we prove Corollary 15 which is the more general result of this paper, and it yields Theorem 2. Finally, we establish, in Corollary 17, certain interesting equivalences among various dynamical notions for $R$-shifts.

\section{SHIFT OPERATORS}

$R$-morphisms between full $R$-shifts. Let $R$ be a PID. $R\left[u, u^{-1}\right]$ denotes the ring of Laurent polynomials with coefficients in $R$. Let $V$ be a finitely generated $R$-module. It is clear that $\sigma$ induces a natural structure of $R\left[u, u^{-1}\right]$-module over $V^{\mathbf{Z}}$ by putting $u \cdot v:=\sigma v$ for all $v \in V^{\mathbf{Z}}$. An $R$-shift over $V$ is then simply a closed $R\left[u, u^{-1}\right]$-submodule of $V^{\mathbf{Z}}$, and $R$-morphisms are the continuous $R\left[u, u^{-1}\right]$ homomorphisms. Hence, a polynomial $p \in R\left[u, u^{-1}\right]$ can be interpreted as an $R$-morphism

$$
p\left(\sigma, \sigma^{-1}\right): R^{\mathbf{Z}} \rightarrow R^{\mathbf{Z}} .
$$

Let $V, W$ be finitely generated $R$-modules. Every $M \in \operatorname{Hom}_{R}(V, W) \otimes R\left[u, u^{-1}\right]$ naturally induces an $R$-morphism

$$
M\left(\sigma, \sigma^{-1}\right): V^{\mathbf{Z}} \rightarrow W^{\mathbf{Z}} .
$$

The morphisms defined in this way are called shift operators. A straightforward consequence of Proposition 1 is that all the $R$-morphisms between full $R$-shifts are given by shift operators.

If $V=R^{q}$ and $W=R^{l}$, we identify $\operatorname{Hom}_{R}(V, W) \otimes R\left[u, u^{-1}\right]$ with $R\left[u, u^{-1}\right]^{l \times q}$ : the $l \times q$ matrices with coefficients in $R\left[u, u^{-1}\right]$. In this case, corresponding shift operators are also called matrix shift operators.

We now need to introduce some more notation. Let $A$ be a unique factorization domain (UFD). $A^{*}$ denotes the multiplicative group of the units of $A$. A multiplicatively closed subset $S \subseteq A(0 \notin S, 1 \in S)$ is said to be saturated if for all $a, b \in A$ we have that $a b \in S \Leftrightarrow a, b \in S$. Let $S \subseteq A$ be a saturated multiplicatively closed set. Define

$$
\tilde{S}:=\left\{a \in A \mid\left(b \notin A^{*} \text { and } b \text { divides } a\right) \Rightarrow b \notin S\right\} .
$$

It is easy to see that $\tilde{S}$ is also a saturated multiplicatively closed set in $A$. An example of a saturated multiplicatively closed subset is $A_{0}:=A \backslash\{0\}$.

Scalar shift operators. Let $p=\sum_{i=l}^{L} p_{i} u^{i} \in R\left[u, u^{-1}\right]$ and suppose that $p_{l}, p_{L}$ are nonzero. If $p_{l}, p_{L} \in R^{*}$ (the ring of units of $R$ ), we say that $p$ is bimonic. Denote by $S_{b}$ the saturated multiplicatively closed subset of all the bimonic polynomials in $R\left[u, u^{-1}\right]$.

We start with a simple preliminary result.

Lemma 3. Let $\mathcal{S} \subseteq R^{\mathbf{Z}}$ be an $R$-shift which is finitely generated as an $R$-module. Then, there exists $p \in S_{b}$ such that $\mathcal{S} \subseteq \operatorname{ker} p\left(\sigma, \sigma^{-1}\right)$.

Proof. Choose $R$-generators $w_{1}, \ldots, w_{n}$ of $\mathcal{S}$ and let $A \in R^{n \times n}$ be such that

$$
\sigma \cdot\left[w_{1} \cdots w_{n}\right]=\left[w_{1} \cdots w_{n}\right] A
$$

Let $p$ be the characteristic polynomial of the matrix $A$. Since $A$ is invertible, $p \in S_{b}$. Let $x \in R^{n}$ and let $w=\left[w_{1} \cdots w_{n}\right] x \in \mathcal{S}$. A straightforward verification shows that

$$
\left(p\left(\sigma, \sigma^{-1}\right) w\right)(t)=\left[w_{1}(0) \cdots w_{n}(0)\right] A^{t} p(A) x=0 \quad \forall t \in \mathbf{Z} .
$$

Hence, $\operatorname{ker} p\left(\sigma, \sigma^{-1}\right) \supseteq \mathcal{S}$. 
Proposition 4. Consider $p \in R\left[u, u^{-1}\right]$. Then, $p\left(\sigma, \sigma^{-1}\right)$ is injective if and only if $p \in \tilde{S}_{b}$

Proof. Suppose that $p \notin \tilde{S}_{b}$. Then, there exists a nontrivial bimonic polynomial $q$ such that $q \mid p$. It is clear that

$$
\operatorname{ker} p\left(\sigma, \sigma^{-1}\right) \supseteq \operatorname{ker} q\left(\sigma, \sigma^{-1}\right) \neq 0
$$

Suppose, conversely, that $p \in \tilde{S}_{b}$. Since $p \neq 0$ it is easy to see that the $R$-shift $\mathcal{S}=\operatorname{ker} p\left(\sigma, \sigma^{-1}\right)$ is finitely generated over $R$. By Lemma 3 there exists a bimonic polynomial $g$ such that $\mathcal{S} \subseteq \operatorname{ker} g\left(\sigma, \sigma^{-1}\right)$. It is clear that $g$ and $p$ must be coprime polynomials, and so there exist $h, k \in R\left[u, u^{-1}\right]$ such that $a=h p+k g \in R_{0}$. Since $a \mathcal{S}=0$, we now have that $\mathcal{S}=0$.

The following gives a characterization of the closure of the image of a shift operator.

Proposition 5. Consider $p \in R\left[u, u^{-1}\right]$ and write $p=a p^{\prime}$ with $a \in R$ and $p^{\prime} \in$ $R\left[u, u^{-1}\right]$ primitive. Then,

$$
\overline{\operatorname{Im} p\left(\sigma, \sigma^{-1}\right)}=a R^{\mathbf{Z}}
$$

Proof. Write $p^{\prime}=\sum_{i=l}^{L} p_{i}^{\prime} u^{i}$. Fix $n \in \mathbf{N}$ and consider the $(2 n+1) \times(2 n+1+L-l)$ matrix

$$
P=\left[\begin{array}{ccccccc}
p_{l}^{\prime} & p_{l+1}^{\prime} & \cdots & p_{L}^{\prime} & 0 & \cdots & 0 \\
0 & p_{l}^{\prime} & \cdots & p_{L-1}^{\prime} & p_{L}^{\prime} & \cdots & 0 \\
& & \ddots & & & & \\
0 & 0 & \cdots & & & \cdots & p_{L}^{\prime}
\end{array}\right]
$$

It is clear that $\operatorname{Im}\left(P: R^{2 n+1+L-l} \rightarrow R^{2 n+1}\right)=\left(\operatorname{Im} p^{\prime}\left(\sigma, \sigma^{-1}\right)\right)_{\mid[-n, n]}$. Elementary considerations of linear algebra over $R$ based on the Smith form [8] show that, since $p_{0}^{\prime}, \ldots, p_{N}^{\prime}$ are coprime, $P$ is surjective, and so $\left(\operatorname{Im} p^{\prime}\left(\sigma, \sigma^{-1}\right)\right)_{\mid[-n, n]}=R^{2 n+1}$. Hence, $\overline{\operatorname{Im} p^{\prime}\left(\sigma, \sigma^{-1}\right)}=R^{\mathbf{Z}}$, which yields the result.

Prime polynomial matrices. We here establish certain primeness results for polynomial matrices which have appeared in a slightly less general form in [12, 14]. We will make use of standard techniques of linear algebra over a PID, essentially based on the so-called Smith form for which the reader is referred to $[8,21,12,14]$.

Let $A$ be a UFD. The rank of $M \in A^{l \times q}$ is defined as the usual rank with respect to the field of fractions of $A . M \in A^{l \times q}$ is said to have full column (resp. row) rank if its rank is equal to $q$ (resp. $l$ ).

$E_{i j} \in A^{l \times q}$ denotes the matrix with all 0's except a 1 at the entry $(i, j) . E(q, A) \subseteq$ $G L(q, A)$ is the subgroup generated by the following elementary matrices:

$$
\begin{gathered}
P_{i j}:=I-E_{i i}-E_{j j}+E_{i j}+E_{j i}, \quad i, j=1, \ldots, q, \\
\Lambda_{i j}(\lambda):=I+\lambda E_{i j}, \quad i \neq j, \quad \lambda \in R .
\end{gathered}
$$

Let $S \subseteq A$ be a multiplicatively closed subset. $M \in A^{l \times q}$ is said to be right $S$-factor prime if it has full column rank and if $M=M^{\prime} F$ with $M^{\prime} \in A^{l \times q}$ and $F \in A^{q \times q}$ yields $\operatorname{det} F \in S$. A matrix $M$ is said to be left $S$-factor prime if $M^{T}$ is right $S$-factor prime. 
Let $R$ be a PID and let $p \in R$ be a prime. Consider the quotient field $k_{p}:=R / p R$. If $M \in R\left[u, u^{-1}\right]^{l \times q}, M_{p} \in k_{p}\left[u, u^{-1}\right]^{l \times q}$ denotes the matrix obtained by reducing modulo $p$ the coefficients of the polynomial entries of $M . M \in R\left[u, u^{-1}\right]^{l \times q}$ is said to be primitive if $M_{p}$ full rank for all prime $p \in R$. We have the following:

Lemma 6. Let $M \in R\left[u, u^{-1}\right]^{l \times q}$ full row rank. Then, there exist $M^{\prime} \in$ $R\left[u, u^{-1}\right]^{l \times l}$ with $\operatorname{det} M^{\prime} \in R_{0}$ and $M^{\prime \prime} \in R\left[u, u^{-1}\right]^{l \times q}$ primitive such that

$$
M=M^{\prime} M^{\prime \prime} \text {. }
$$

Proof. If $M$ is not primitive, let $p \in R$ be a prime such that $M_{p} \in k_{p}\left[u, u^{-1}\right]^{l \times q}$ does not have full row rank. Using standard techniques based on the Smith form on the PID $k_{p}\left[u, u^{-1}\right]$, we can find $\bar{U} \in E\left(l, k_{p}\left[u, u^{-1}\right]\right)$ such that

$$
\bar{U} M_{p}=\left[\begin{array}{c}
\bar{X} \\
0
\end{array}\right],
$$

where $\bar{X} \in k_{p}\left[u, u^{-1}\right]^{l-1 \times q}$. Let $U \in E\left(l, R\left[u, u^{-1}\right]\right)$ be such that $U_{p}=\bar{U}$. It follows that

$$
M=U^{-1}\left[\begin{array}{ll}
I & 0 \\
0 & p
\end{array}\right]\left[\begin{array}{l}
X \\
Y
\end{array}\right],
$$

where $X \in R\left[u, u^{-1}\right]^{l-1 \times q}$ and $Y \in R\left[u, u^{-1}\right]^{1 \times q}$. We conclude by induction.

Theorem 7. Let $S \subseteq R\left[u, u^{-1}\right]$ be a saturated multiplicatively closed set such that $S^{-1} R\left[u, u^{-1}\right]$ is a PID. Let $M \in R\left[u, u^{-1}\right]^{l \times q}$ have full column rank. Then, the following facts are equivalent:

1. $M$ is right $S$-factor prime.

2. Every common factor of the $q \times q$ minors of $M$ is in $S$.

3. There exist $X \in R\left[u, u^{-1}\right]^{q \times l}$ and $a \in S$ such that

$$
X M=a I .
$$

Proof. $(1 \Rightarrow 2)$ Factor $M=M^{\prime} \Lambda$, where $M^{\prime} \in R\left[u, u^{-1}\right]^{l \times q}$ is right $R\left[u, u^{-1}\right]^{*}$ factor prime and $\Lambda \in R\left[u, u^{-1}\right]^{q \times q}$ is such that $\operatorname{det} \Lambda \in S$. It is sufficient to prove that all the common factors of the $q \times q$ minors of $M^{\prime}$ are in $R\left[u, u^{-1}\right]^{*}$. Let $F$ be the field of fractions of $R$. We now show that $M^{\prime}$ is right $F\left[u, u^{-1}\right]^{*}$-factor prime as a matrix in $F\left[u, u^{-1}\right]^{l \times q}$. Suppose it is not. Then, elementary considerations based on the Smith form on the PID $F\left[u, u^{-1}\right]$ show that there exist $U \in R\left[u, u^{-1}\right]^{l \times l}$ with $\operatorname{det} U \in R_{0}$ and $A \in R\left[u, u^{-1}\right]^{q \times q}$ with $\operatorname{det} A$ not a monomial, such that

$$
U M^{\prime}=\left[\begin{array}{c}
A \\
0
\end{array}\right] \text {. }
$$

Factor $A=A^{\prime} A^{\prime \prime}$, where $A^{\prime} \in R\left[u, u^{-1}\right]^{q \times q}$ with $\operatorname{det} A^{\prime} \in R_{0}$ and $A^{\prime \prime} \in R\left[u, u^{-1}\right]^{q \times q}$ is primitive, according to Lemma 6 . Now, if $a:=\operatorname{det} U$ and $\left[\tilde{U}_{1} \tilde{U}_{2}\right]:=\operatorname{Adj}(U)$, then we have that

$$
a M^{\prime}=\left[\begin{array}{ll}
\tilde{U}_{1} & \tilde{U}_{2}
\end{array}\right]\left[\begin{array}{c}
A^{\prime} A^{\prime \prime} \\
0
\end{array}\right]=\tilde{U}_{1} A^{\prime} A^{\prime \prime} .
$$

Since $A^{\prime \prime}$ is primitive, we have that $M^{\prime}=B A^{\prime \prime}$ for some $B \in R\left[u, u^{-1}\right]^{l \times q}$. This contradicts the fact that $M^{\prime}$ is right $R\left[u, u^{-1}\right]^{*}$-factor prime. This proves our claim, which implies that all common factors of the $q \times q$ minors of $M^{\prime}$ are of the type 
$a u^{k}$ for some $a \in R_{0}$ and $k \in \mathbf{Z}$. However, since $M^{\prime}$ is primitive by construction, it follows that $a \in R^{*}$ necessarily. This completes the proof.

$(2 \Rightarrow 3)$ Let $m_{1}, m_{2}, \ldots, m_{s}$ be the $q \times q$ minors of $M$. Since $S^{-1} R\left[u, u^{-1}\right]$ is a PID, there exist $h_{1}, h_{2}, \ldots, h_{s} \in R\left[u, u^{-1}\right]$ such that

$$
\sum_{i=1}^{s} h_{i} m_{i}=a \in S
$$

Let $S_{i} \in R^{q \times l}$ be such that $m_{i}=\operatorname{det}\left(S_{i} M\right)$. Then,

$$
a I=\sum_{i=1}^{s} h_{i} m_{i} I=\sum_{i=1}^{s} h_{i}\left(\operatorname{det} S_{i} M\right) I=\left[\sum_{i=1}^{s} h_{i} \operatorname{Adj}\left(S_{i} M\right) S_{i}\right] M .
$$

$(3 \Rightarrow 1)$ is straightforward.

\section{Matrix shift operators.}

Theorem 8. Let $M \in R\left[u, u^{-1}\right]^{l \times q}$. Then, the following facts are equivalent:

1. $M\left(\sigma, \sigma^{-1}\right)$ is injective.

2. There exist $X \in R\left[u, u^{-1}\right]^{q \times l}$ and $a \in \tilde{S}_{b}$ such that

$$
X M=a I .
$$

3. $M$ is right $\tilde{S}_{b}$-factor prime.

Proof. $(3 \Rightarrow 2)$ is a consequence of Theorem 7 , considering the fact that, since $R_{0} \subseteq$ $\tilde{S}_{b}, \tilde{S}_{b}^{-1} R\left[u, u^{-1}\right]$ is a PID.

$(2 \Rightarrow 1)$ is a consequence of Proposition 4 .

$(1 \Rightarrow 3) M$ has full column rank, because otherwise it would be possible to find a nonzero sequence with finite support in the kernel of $M\left(\sigma, \sigma^{-1}\right)$. Consider the factorization $M=M^{\prime} \Lambda$, where $M^{\prime} \in R\left[u, u^{-1}\right]^{l \times q}$ and $\Lambda \in R\left[u, u^{-1}\right]^{q \times q}$. By standard Smith form techniques on the PID $F\left[u, u^{-1}\right]$, where $F$ is the field of fractions of $R$, we can find $U, V \in R\left[u, u^{-1}\right]^{q \times q}$ with $\operatorname{det} U$, $\operatorname{det} V \in R_{0}$ such that $U \Lambda V=D$, where $D$ is a diagonal matrix in $R\left[u, u^{-1}\right]^{q \times q}$ whose diagonal elements are different from zero. It easily follows from Proposition 4 that $U\left(\sigma, \sigma^{-1}\right)$ and $V\left(\sigma, \sigma^{-1}\right)$ are injective shift operators, and so also $D\left(\sigma, \sigma^{-1}\right)$ is injective. Since $D$ is diagonal, we have, by Proposition 4 , that $\operatorname{det} D \in \tilde{S}_{b}$. This yields $\operatorname{det} \Lambda \in \tilde{S}_{b}$.

We now give a characterization of the closure of the image of a shift operator.

Proposition 9. Let $M \in R\left[u, u^{-1}\right]^{l \times q}$ have full column rank. Then, there exists a right $R_{0}\left(R\left[u, u^{-1}\right]\right)^{*}$-factor prime $M_{1} \in R\left[u, u^{-1}\right]^{l \times q}$ such that

$$
\overline{\operatorname{Im} M\left(\sigma, \sigma^{-1}\right)}=\operatorname{Im} M_{1}\left(\sigma, \sigma^{-1}\right),
$$

Proof. Factor $M=\tilde{M} \Lambda$ with $\tilde{M} \in R\left[u, u^{-1}\right]^{l \times q}$ right $\left(R\left[u, u^{-1}\right]\right)^{*}$-factor prime and $\Lambda \in R\left[u, u^{-1}\right]^{q \times q}$. By Lemma 6 there exist $\Lambda_{1}, \Lambda_{2} \in R\left[u, u^{-1}\right]^{q \times q}$ with $\operatorname{det} \Lambda_{1} \in$ $R_{0}$ and $\Lambda_{2}$ primitive, such that $\Lambda=\Lambda_{1} \Lambda_{2}$. Put $M_{1}:=\tilde{M} \Lambda_{1}$ and $M_{2}:=\Lambda_{2}$. Since, by Theorem $7, M_{1}\left(\sigma, \sigma^{-1}\right)$ admits a continuous inverse on its image, then $\operatorname{Im} M_{1}\left(\sigma, \sigma^{-1}\right)$ is closed. Consequently,

$$
\overline{\operatorname{Im} M\left(\sigma, \sigma^{-1}\right)} \subseteq \operatorname{Im} M_{1}\left(\sigma, \sigma^{-1}\right) .
$$

On the other hand,

$$
\overline{\operatorname{Im} M\left(\sigma, \sigma^{-1}\right)} \supseteq M_{1}\left(\sigma, \sigma^{-1}\right)\left(\overline{\operatorname{Im} M_{2}\left(\sigma, \sigma^{-1}\right)}\right)=\operatorname{Im} M_{1}\left(\sigma, \sigma^{-1}\right),
$$

where the last equality follows from Proposition 5 applied to $\operatorname{det} M_{2}$. 


\section{Transitive Finite MEMORY $R$-SHIFtS}

Controllable and weakly controllable $R$-shifts. We now introduce a concept which is in general stronger than topological transitivity. It comes from the behavioral approach to systems theory [21] and has already been used in symbolic dynamics in [6]. As always, let $R$ be a PID and $V$ a finitely generated $R$-module. Let $\mathcal{S} \subseteq V^{\mathbf{Z}}$ be an $R$-shift. $\mathcal{S}$ is said to be controllable if for all $v_{1}, v_{2} \in \mathcal{S}$, there exist $n \in \mathbf{N}$ and $v \in \mathcal{S}$ with

$$
v(t)=v_{1}(t) \forall t<0, \quad\left(\sigma^{n} v\right)(t)=v_{2}(t) \forall t \geq 0 .
$$

Let $\mathcal{S}$ be an $R$-shift and let $\mathcal{S}_{f}$ be the $R\left[u, u^{-1}\right]$-submodule of $\mathcal{S}$ consisting of finite support sequences in $\mathcal{S}$. $\mathcal{S}$ is called weakly controllable if $\overline{\mathcal{S}_{f}}=\mathcal{S}$. It is easy to see that controllability implies weak controllability, which in turn implies transitivity. Moreover, since $V$ is Noetherian, every controllable $\mathcal{S}$ over $V$ satisfies a stronger notion of controllability [22] in the sense that the number $n \in \mathbf{N}$ in (28) can be chosen a priori for all pairs $v_{1}, v_{2} \in \mathcal{S}$ : from this it follows that controllability also implies topological mixing. On the other hand, it is easy to see that if we have finite memory, the four properties coincide: in particular transitive finite memory $R$-shifts are controllable. It is easy to see, using the techniques of [6], that also controllability and weak controllability are preserved by $R$-conjugacies.

The free case. We now apply the results of Section 2 to characterize weakly controllable $R$-shifts over finitely generated free $R$-modules.

Theorem 10. Let $R$ be a PID and let $\mathcal{S} \subseteq\left(R^{l}\right)^{\mathbf{Z}}$ be a weakly controllable $R$-shift. Then, there exists a $q \in \mathbf{N}$ such that $\mathcal{S} \simeq_{R}\left(R^{q}\right)^{\mathbf{Z}}$.

Proof. Since $\mathcal{S}_{f}$ is a finitely generated $R\left[u, u^{-1}\right]$-module, there exist $q^{\prime} \in \mathbf{N}$ and $N \in R\left[u, u^{-1}\right]^{l \times q^{\prime}}$ such that

$$
\mathcal{S}_{f}=N\left(\sigma, \sigma^{-1}\right)(\mathcal{F})
$$

where $\mathcal{F}:=\left(R^{q^{\prime}}\right)_{f}^{\mathbf{Z}}$. Then,

$$
\mathcal{S}=\overline{\mathcal{S}_{f}}=\overline{\operatorname{Im} N\left(\sigma, \sigma^{-1}\right)}
$$

Consider now the $\operatorname{ring} A=S_{b}^{-1} R\left[u, u^{-1}\right]$. Using techniques similar to [13] (Chapter IV), it can be shown that $A$ is a PID. Using standard Smith forms techniques on the PID $A$, we can find $p \in S_{b}$, matrices $U \in R\left[u, u^{-1}\right]^{l \times l}, V \in R\left[u, u^{-1}\right]^{q^{\prime} \times q^{\prime}}$ with $\operatorname{det} U$, det $V \in S_{b}$, and a diagonal non-singular $D \in R\left[u, u^{-1}\right]^{q \times q}$ such that

$$
p N=U\left[\begin{array}{cc}
D & 0 \\
0 & 0
\end{array}\right] V .
$$

Since $V\left(\sigma, \sigma^{-1}\right)$ and $p\left(\sigma, \sigma^{-1}\right)$ are clearly surjective, then

$$
\operatorname{Im} N\left(\sigma, \sigma^{-1}\right)=\operatorname{Im} N^{\prime}\left(\sigma, \sigma^{-1}\right),
$$

where

$$
N^{\prime}:=U\left[\begin{array}{c}
D \\
0
\end{array}\right]
$$

now has full column rank. By Proposition 9 , there exists a right $R_{0}\left(R\left[u, u^{-1}\right]\right)^{*}$ factor prime $M \in R\left[u, u^{-1}\right]^{l \times q}$ such that

$$
\mathcal{S}=\operatorname{Im} M\left(\sigma, \sigma^{-1}\right) \text {. }
$$


$M\left(\sigma, \sigma^{-1}\right)$ is injective by Theorem 8, and it follows from Theorem 7 that the inverse of $M\left(\sigma, \sigma^{-1}\right)$ on the image $\mathcal{S}$ is continuous. Therefore, $M\left(\sigma, \sigma^{-1}\right)$ is an $R$-conjugacy between $\left(R^{q}\right)^{\mathbf{Z}}$ and $\mathcal{S}$.

Remark. Since transitive finite memory $R$-shifts are weakly controllable, Theorem 10 yields Theorem 2 in the case when the alphabet $V$ is a free $R$-module.

Remark. Note that Theorem 10 yields the surprising fact that a weakly controllable $R$-shift $\mathcal{S} \subseteq\left(R^{l}\right)^{\mathbf{Z}}$ automatically has finite memory and is controllable. This will be generalized in the sequel.

The torsion case. We now study transitive $R$-shifts over finitely generated torsion $R$-modules, generalizing certain results which appeared in [5] in the case $R=$ Z. Note first that since finitely generated torsion $R$-modules are Artinian, such $R$-shifts always have finite memory [22]. As a consequence, transitivity, mixing, controllability, and weak controllability coincide in this context.

If $E$ is any $R$-module and $a \in R$, define the $R$-module

$$
E_{(a)}:=\{x \in E \mid a x=0\} .
$$

Moreover, define $\tau(E)=\{a \in R \mid a E=0\}$ and let $t(E)$ be a generator of $\tau(E)$.

Let $V$ be a finitely generated $R$-module. If $\mathcal{S} \subseteq V^{\mathbf{Z}}$ is an $R$-shift and $a \in R$, then $\mathcal{S}_{(a)}$ is also an $R$-shift. Moreover, if $V$ is $R$-torsion, we can write $t(\mathcal{S})=t=$ $p_{1}^{r_{1}} \cdots p_{k}^{r_{k}}$ (the $p_{i} \in R$ are distinct primes, and $r_{i} \in \mathbf{N}$ ), and we have that

$$
\mathcal{S}=\bigoplus_{i=1}^{k} \mathcal{S}_{\left(p_{i}^{r_{i}}\right)}
$$

It is easy to see that $\mathcal{S}$ is transitive if and only if each $\mathcal{S}_{\left(p_{i}^{r_{i}}\right)}$ is transitive.

If $p \in R$ is a prime and $n \in \mathbf{N}$, then $A:=R / p^{n} R$ is a complete local Artinian ring with maximal ideal $m=p R / p^{n} R$ and residue field $k=R / p R$. Notice moreover that the $m$-adic topology on $A$ coincides with the discrete topology. Let $V$ be a finitely generated $A$-module with the discrete topology. Since $V$ is also Artinian, it follows that $V$ is linearly compact [23]. This implies that also the product space $V^{\mathbf{Z}}$ is linearly compact. Hence every $A$-shift $\mathcal{S} \subseteq V^{\mathbf{Z}}$ is linearly compact. A useful tool in this context is the duality theory over complete local rings, for which we refer the reader to [15]. In this case, duality theory is particularly simple since $A$ itself is the injective envelope of $k$. If $E$ is a linearly topologized $A$-module (i.e. a topological $A$-module for which there exists a basis of neighborhoods of 0 consisting of open submodules), denote by $E^{*}:=\operatorname{Hom}_{A}(E, A)$ the $A$-module of continuous $A$-homomorphisms from $E$ to $A$ topologized with the usual compact-open topology. As it happens for the Pontrjagin duality, $E \mapsto E^{*}$ then yields an exact contravariant functorial correspondence between the category of linearly compact $A$-modules and the category of discrete $A$-modules.

Theorem 11. Let $R$ be a PID and let $V$ be a finitely generated torsion $R$-module. Consider an $R$-shift $\mathcal{S} \subseteq V^{\mathbf{Z}}$ with $t(\mathcal{S})=p_{1}^{r_{1}} \cdots p_{k}^{r_{k}}$ (the $p_{i} \in R$ are distinct primes, and $\left.r_{i} \in \mathbf{N}\right)$. The following conditions are equivalent:

1. There exists a finitely generated torsion $R$-module $H$ such that $\mathcal{S} \simeq_{R} H^{\mathbf{Z}}$.

2. $\mathcal{S}_{(r)}$ is transitive for all $r \in R$.

3. $\mathcal{S}_{\left(p_{i}^{j}\right)}$ is transitive for all $i=1, \ldots, k$ and $j=1, \ldots, r_{i}$.

4. $p_{i}^{j-1} \mathcal{S}_{\left(p_{i}^{j}\right)}$ is transitive for all $i=1, \ldots, k$ and $j=1, \ldots, r_{i}$. 
Proof. $(1 \Rightarrow 2 \Rightarrow 3 \Rightarrow 4)$ is evident.

$(4 \Rightarrow 1)$ By virtue of previous considerations $(36)$ it is sufficient to prove it in the case $k=1$. In this case we can also assume that $V$ is a module over $A=R / p^{r} R$. We can therefore apply the duality theory and repeat the same proof as in the case $R=\mathbf{Z}$ presented in [5].

The general case. We now want to combine Theorems 10 and 11 to get a more general result. We first need to establish a few preliminary results.

Let $V$ be a finitely generated $R$-module. Factor $V=F \oplus T$, where $F$ is a free $R$-module and $T=T(V)$ is the torsion submodule of $V$. Let $\mathcal{S} \subseteq V^{\mathbf{Z}}$ be an $R$-shift. The $R$-torsion submodule of $\mathcal{S}, T(\mathcal{S})=\mathcal{S} \cap T^{\mathbf{Z}}$, is clearly an $R$-shift. Define $\mathcal{S}_{F}$ to be the projection of $\mathcal{S}$ on $F^{\mathbf{Z}}$. We start with the following.

Lemma 12. For all $k \in \mathbf{N}$ there exists $n_{k} \in \mathbf{N}\left(n_{k} \geq k\right)$ such that

$$
T\left(\mathcal{S}_{\mid\left[-n_{k}, n_{k}\right]}\right)_{\mid[-k, k]}=T(\mathcal{S})_{\mid[-k, k]} .
$$

Proof. $\supseteq$ in (37) is evident. Fix $k \in \mathbf{N}$ and notice that as $m$ varies, $T\left(\mathcal{S}_{\mid[-m, m]}\right)_{\mid[-k, k]}$ form a descending chain of $R$-submodules inside $T^{2 k+1}$. Hence, there exist $n_{k} \in \mathbf{N}$ such that

$$
T\left(\mathcal{S}_{\mid[-m, m]}\right)_{\mid[-k, k]}=T\left(\mathcal{S}_{\mid\left[-n_{k}, n_{k}\right]}\right)_{\mid[-k, k]} \quad \forall m \geq n_{k} .
$$

Fix $v \in T\left(\mathcal{S}_{\mid\left[-n_{k}, n_{k}\right]}\right)_{\mid[-k, k]}$. It is easy to see that there exists a sequence $v_{i} \in$ $T\left(\mathcal{S}_{\mid\left[-n_{i}, n_{i}\right]}\right)_{\mid[-i, i]}$ for $i \geq k$ such that $v_{k}=v$ and

$$
\left.v_{i+1}\right|_{[-i, i]}=v_{i} \quad \forall i \geq k .
$$

Now let $\tilde{v}_{i} \in \mathcal{S}$ be such that $\tilde{v}_{i \mid[-i, i]}=v_{i}$ for all $i \geq k$. Clearly, $\tilde{v}_{i} \rightarrow \tilde{v}$ for some $\tilde{v} \in T(\mathcal{S})$. We have that $\tilde{v}_{\mid[-k, k]}=v$, and this completes the proof.

We can now prove the following.

Proposition 13. $\mathcal{S}_{F}$ is an R-shift.

Proof. We only need to show that $\mathcal{S}_{F}$ is closed. Let $f_{n} \in \mathcal{S}_{F}$ be such that $f_{n} \rightarrow f \in$ $F^{\mathbf{Z}}$. To prove that $f \in \mathcal{S}_{F}$ it is clearly not restrictive to assume that $f_{n \mid[-n, n]}=$ $f_{[[-n, n]}$. We claim that there exists a sequence $g_{n} \in T^{\mathbf{Z}}$ such that $f_{n}+g_{n} \in \mathcal{S}$ for all $n \in \mathbf{N}$ and

$$
g_{n \mid[-k, k]}=g_{n_{k} \mid[-k, k]} \quad \forall k \in \mathbf{N} \quad \forall n \geq n_{k},
$$

where $n_{k}$ is defined as in Lemma 12 and in such a way that $n_{k+1} \geq n_{k}$ for all $k$. Indeed, fix $g_{n_{0}}$ arbitrarily such that $f_{n_{0}}+g_{n_{0}} \in \mathcal{S}$. By induction, suppose we have constructed $g_{n}$ with the desired properties for $n \leq n_{h}+s<n_{h+1}$. Choose $g^{\prime} \in T^{\mathbf{Z}}$ such that $f_{n_{h}+s+1}+g^{\prime} \in \mathcal{S}$. Notice that

$$
\tilde{g}:=g_{\mid\left[-n_{h}, n_{h}\right]}^{\prime}-\left(g_{n_{h}+s}\right)_{\mid\left[-n_{h}, n_{h}\right]} \in T\left(\mathcal{S}_{\mid\left[-n_{h}, n_{h}\right]}\right) .
$$

Hence,

$$
\tilde{g}_{\mid[-h, h]} \in T(\mathcal{S})_{\mid[-h, h]} .
$$

Let $g^{\prime \prime} \in T(\mathcal{S})$ be such that $g_{\mid[-h, h]}^{\prime \prime}=\tilde{g}_{\mid[-h, h]}$. Define $g_{n_{h}+s+1}:=g^{\prime}-g^{\prime \prime}$. It is easy to verify that the sequence $g_{n}$ defined in this way satisfies the required properties. It is also clear that $g_{n} \rightarrow g \in T^{\mathbf{Z}}$. Hence $f \in \mathcal{S}_{F}$.

Finally, we have the following key fact. 
Theorem 14. If $T(\mathcal{S}) \simeq_{R} H^{\mathbf{Z}}$ for some R-torsion module $H$, then

$$
\mathcal{S} \simeq_{R} \mathcal{S}_{F} \oplus T(\mathcal{S}) .
$$

Proof. Let $\phi: T(\mathcal{S}) \rightarrow H^{\mathbf{Z}}$ be an $R$-conjugacy with memory 0 . Since $T(\mathcal{S})$ has finite memory and $\phi$ has the structure described in Proposition 1, by taking a suitable block extension [9] $\tilde{T}:=\left(T^{\mathbf{Z}}\right)^{[n]} \subseteq\left(T^{n}\right)^{\mathbf{Z}}$, we have that $T(\mathcal{S})^{[n]}=T^{\prime \mathbf{Z}} \cap \tilde{T}$, where $T^{\prime}:=T(\mathcal{S})_{\mid[0, n-1]}$. Moreover, we have an $R$-homomorphism

$$
\zeta: T^{\prime} \rightarrow H
$$

which induces an $R$-conjugacy

$$
\zeta^{\infty}: T(\mathcal{S})^{[n]} \rightarrow H^{\mathbf{Z}}
$$

The block extension on $T^{\mathbf{Z}}$ induces an obvious $R$-conjugacy from $F^{\mathbf{Z}} \oplus T^{\mathbf{Z}}$ to $F^{\mathbf{Z}} \oplus \tilde{T} \subseteq F^{\mathbf{Z}} \oplus\left(T^{n}\right)^{\mathbf{Z}}$. Therefore, it is not restrictive to assume that $\mathcal{S} \subseteq F^{\mathbf{Z}} \oplus \tilde{T}$, where $\tilde{T} \subseteq T^{\mathbf{Z}}$ is an $R$-shift, and that there exist an $R$-submodule $T^{\prime} \subseteq T$ and an $R$-homomorphism $\zeta$ as in (44) such that $T(\mathcal{S})=T^{\prime \mathbf{Z}} \cap \tilde{T}$ and

$$
\zeta^{\infty}: T(\mathcal{S}) \rightarrow H^{\mathbf{Z}}
$$

is an $R$-conjugacy. Let $K=\operatorname{ker} \zeta$ and consider the quotient projection $p: T \rightarrow$ $T / K$. Notice that

$$
\tilde{T} \cap K^{\mathbf{Z}}=\left(\tilde{T} \cap T^{\prime \mathbf{Z}}\right) \cap K^{\mathbf{Z}}=T(\mathcal{S}) \cap K^{\mathbf{Z}}=\{0\} .
$$

Hence $p^{\infty}$ induces an $R$-conjugacy between $\tilde{T}$ and $S:=p^{\infty}(\tilde{T}) \subseteq(T / K)^{\mathbf{Z}}$. Now let $\eta: H \rightarrow T / K$ be the injective $R$-homomorphism such that $\eta \zeta=p_{\mid T^{\prime}}$. Hence

$$
p^{\infty}(T(\mathcal{S}))=\eta^{\infty}\left(H^{\mathbf{Z}}\right)=\tilde{H}^{\mathbf{Z}},
$$

where $\tilde{H}:=\eta(H)$. By applying the $R$-conjugacy from $\tilde{T}$ to $S$, we can therefore assume that $\mathcal{S} \subseteq(F \oplus T)^{\mathbf{Z}}$ and $T(\mathcal{S})=H^{\mathbf{z}}$, where $H$ is an $R$-submodule of $T$. Denote by $\pi: T \rightarrow T / H$ the quotient map. Define

$$
\Gamma: \mathcal{S}_{F} \rightarrow(T / H)^{\mathbf{Z}}
$$

by

$$
\Gamma(f)=\pi(g)
$$

where $g \in T^{\mathbf{Z}}$ is such that $f+g \in \mathcal{S}$. $\Gamma$ is a well-defined $R\left[u, u^{-1}\right]$-homomorphism, and it easily follows from Lemma 12 that it is continuous. Hence, by Proposition 1 , there exists an $R$-homomorphism

$$
\gamma: \mathcal{S}_{F \mid[0, N+M]} \rightarrow T / H
$$

such that

$$
(\Gamma f)(t)=\gamma\left(f_{\mid[t-N, t+M]}\right) \quad \forall t \in \mathbf{Z} .
$$

Consider now a lifting of $\gamma$ :

$$
\delta: \mathcal{S}_{F \mid[0, N+M]} \rightarrow T .
$$

$\delta$ induces a lifting of $\Gamma$

$$
\Delta: \mathcal{S}_{F} \rightarrow T^{\mathbf{Z}} .
$$

Consider

$$
\psi: \mathcal{S}_{F} \oplus H^{\mathbf{Z}} \rightarrow \mathcal{S}
$$


given by

$$
\psi(f, h):=f+\Delta f+h .
$$

$\psi$ is a well defined $R$-morphism. Moreover, it is invertible and the inverse, as can easily be checked, is given by

$$
\psi^{-1}: \mathcal{S} \rightarrow \mathcal{S}_{F} \oplus H^{\mathbf{Z}}
$$

with

$$
\psi^{-1}(v)=(f, g-\Delta f),
$$

where $f$ and $g$ denote, respectively, the projection of $v$ on $F^{\mathbf{Z}}$ and on $T^{\mathbf{Z}}$. Clearly, also $\psi^{-1}$ is continuous, and the proof is thus complete.

Corollary 15. Let $R$ be a PID and $V$ a finitely generated $R$-module. Let $\mathcal{S} \subseteq V^{\mathbf{Z}}$ be an $R$-shift. The following conditions are equivalent:

1. $\mathcal{S} \simeq_{R} H^{\mathbf{Z}}$ for some $R$-module $H$.

2. $\mathcal{S}_{(r)}$ is controllable for all $r \in R$.

3. $\mathcal{S}_{(r)}$ is weakly controllable for all $r \in R$.

4. $\mathcal{S}_{(r)}$ is transitive and has finite memory for all $r \in R$.

Proof. $(1 . \Rightarrow 2 . \Rightarrow 3 . \Rightarrow 4$. $)$ is evident.

$\left(4 . \Rightarrow 1\right.$.) Since $T(\mathcal{S})_{(r)}=\mathcal{S}_{(r)}$ if $r \neq 0$, it follows from Theorem 11 that $T(\mathcal{S})$ is $R$-conjugated to a full $R$-shift. We can therefore apply Theorem 14 and conclude by using Theorem 10, after noticing that $\mathcal{S}_{F} \subseteq F^{\mathbf{Z}}$ is transitive and has finite memory.

Proof of Theorem 2. Theorem 2 is an immediate consequence of Corollary 15: if $\mathcal{S}$ is $R$-torsion free, then $\mathcal{S}_{(r)}=0$ if $r \in R_{0}$, and $\mathcal{S}_{(0)}=\mathcal{S}$.

A final result. In the notation of the previous subsection, we have the following.

Proposition 16. $\mathcal{S}$ has finite memory if and only if $\mathcal{S}_{F}$ has finite memory.

Proof. "If": Let $N \in \mathbf{N}$ be such that both $\mathcal{S}_{F}$ and $T(\mathcal{S})$ have memory $N$. Let $s \in \mathbf{N}$ be such that

$$
T\left(\mathcal{S}_{\mid[-s, N+s]}\right)_{\mid[0, N]}=T(\mathcal{S})_{\mid[0, N]} .
$$

We know that such an $s$ exists by Lemma 12. Put $M=N+2 s$. We now prove that $\mathcal{S}$ has memory $M$. Let $v=f+g$ with $f \in F^{\mathbf{Z}}$ and $g \in T^{\mathbf{Z}}$ be such that

$$
v_{\mid[t, t+M]} \in \mathcal{S}_{\mid[0, M]} \quad \forall t \in \mathbf{Z} .
$$

It follows that

$$
f_{\mid[t, t+M]} \in \mathcal{S}_{F \mid[0, M]} \quad \forall t \in \mathbf{Z} .
$$

Hence, there exists $\tilde{g} \in T^{\mathbf{Z}}$ such that $\tilde{v}=f+\tilde{g} \in \mathcal{S}$. Consider $z:=v-\tilde{v}=g-\tilde{g}$. It is clear that

$$
z_{\mid[t, t+M]} \in T\left(\mathcal{S}_{\mid[0, M]}\right) \quad \forall t \in \mathbf{Z}
$$

and, by (59),

$$
z_{\mid[t, t+N]} \in T(\mathcal{S})_{\mid[0, N]} \quad \forall t \in \mathbf{Z} .
$$

Since $T(\mathcal{S})$ has memory $N$, it then follows that $z=v-\tilde{v} \in T(\mathcal{S})$, and this yields $v \in \mathcal{S}$. 
"Only if": Let $N \in \mathbf{N}$ be such that $\mathcal{S}$ has memory $N$. Let $s \in \mathbf{N}$ be such that (59) holds true with respect to $N-1$. We now prove that $\mathcal{S}_{F}$ has memory $M=N+2 s$. Let $f \in \mathcal{S}_{F}$ be such that $f_{\mid[0, M)}=0$ and let $g \in T^{\mathbf{Z}}$ be such that $v:=f+g \in \mathcal{S}$. By the way we have chosen $M$, there exists $\tilde{g} \in T(\mathcal{S})$ such that $\tilde{g}_{\mid[s, M-s)}=g_{\mid[s, M-s)}$. Consider now

$$
\tilde{v}:=v-\tilde{g}=f+g-\tilde{g} \in \mathcal{S} .
$$

Notice that $\tilde{v}_{\mid[s, M-s)}=0$. Hence, there exists $w \in \mathcal{S}$ such that $w_{\mid[0,+\infty)}=v_{\mid[0,+\infty)}$ and $w_{\mid(-\infty, 0)}=0$. Denote by $f^{\prime}$ the projection of $w$ on $F^{\mathbf{Z}}$. Clearly, $f_{\mid[0,+\infty)}^{\prime}=$ $f_{\mid[0,+\infty)}$ and $f_{\mid(-\infty, 0)}^{\prime}=0$, which proves the result.

Finally, we have the following.

Corollary 17. Let $R$ be a PID and let $V$ be a finitely generated $R$-module. Let $\mathcal{S} \subseteq V^{\mathbf{Z}}$ be an $R$-shift. The following conditions are equivalent:

1. $\mathcal{S}$ is controllable.

2. $\mathcal{S}$ is weakly controllable.

3. $\mathcal{S}$ is transitive and has finite memory.

4. $\mathcal{S}$ is mixing and has finite memory.

Proof. We only need to prove that if $\mathcal{S}$ is weakly controllable, then it has finite memory. Write $V=F \oplus T$, where $F$ is a free $R$-module and $T=T(V)$, and consider the exact sequence

$$
0 \rightarrow T(\mathcal{S}) \hookrightarrow \mathcal{S} \rightarrow \mathcal{S}_{F} \rightarrow 0 .
$$

Clearly, $\mathcal{S}_{F}$ is weakly controllable and, hence, controllable and finite memory by Theorem 10 and the remark following it. It then follows from Proposition 16 that $\mathcal{S}$ has finite memory.

\section{REFERENCES}

[1] F. Blanchard. Systèmes dynamiques topologiques associés à des automates récurrents. Z. Wahr. Verw. Geb., 58 (1981), 549-564. MR 83c:68101

[2] F. Blanchard, G. Hansel. Systèmes codés. Theoretical Computer Science, 44 (1986), 17-49. MR 87i: 93002

[3] W. Brewer, J.W. Bunce, and F.S. Van Vleck. Linear systems over commutative rings. Dekker, 1986.

[4] E.E. Dinaburg. An example of the computation of topological entropy. Uspehi Mat. Nauk., 23 (1968), 249-250. MR 37:5883

[5] F. Fagnani. Some results on the classification of expansive automorphisms of compact Abelian groups. Ergodic Theory and Dynamical Systems, 16 (1996), 45-50. CMP 96:8

[6] F. Fagnani. Shifts on compact and discrete Lie groups: algebraic-topological invariants and classification problems. To appear in Advances in Mathematics.

[7] G.D. Forney and M.D. Trott. The dynamics of group codes: State spaces, trellis diagrams and canonical encoders. IEEE Trans. on Information Theory, IT-39 (1993), 1491-1513. MR 96e: 94017

[8] P. Fuhrmann Linear systems and operators in Hilbert spaces. McGraw-Hill, New York, 1981. MR 83d: 47001

[9] B.P. Kitchens. Expansive dynamics on zero-dimensional groups. Ergodic Theory and Dynamical Systems, 7 (1987), 249-261. MR 88i:28039

[10] B.P. Kitchens and K. Schmidt. Automorphisms of compact groups. Ergodic Theory and Dynamical Systems, 9 (1989), 691-735. MR 91g:22008

[11] W. Krieger. On the uniqueness of the equilibrium state. Math. Systems Theory, 8 (1974), 97-104. MR 53:3256 
[12] S. Kung, B. Lévy, M. Morf, and T. Kailath. New results in $2 D$ systems theory. Part 1. Proc. IEEE, 65 (1977), 861-872.

[13] T.Y Lam, Serre's conjecture Springer LNM 635, 1978. MR 58:5644

[14] B. Lévy. 2D polynomial and rational matrices, and their applications for the modelling of 2D dynamical systems. Technical Report M735-11, Stanford Electronics Lab., 1981.

[15] I.G. MacDonald. Duality over complete local rings. Topology, 1 (1962), 213-235. MR 27:1476

[16] G. Miles and R.K. Thomas. The breakdown of automorphisms of compact topological groups. In G.C. Rota, editor, Studies in Probability and Ergodic Theory, 207-218. Academic Press, 1978. MR 80c:22007

[17] K. Petersen. Chains, entropy, coding. Ergodic Theory Dynamical Systems, 6 (1986), 415-448. MR 88i: 28040

[18] I.A. Salama. Topological entropy and recurrence of countable chains. Pacific J. Math., 134 (1988), 325-341; errata: Pacific J. Math., 140 (1989), 397-398. MR 90d:54076; MR 90k:54055

[19] K. Schmidt. Automorphisms of compact Abelian groups and affine varieties. Proc. London Math. Soc. 61 (1990), 480-496. MR 91j:28015

[20] E.D. Sontag. Linear systems over commutative rings: A survey. Ricerche di Automatica, 7 (1976), 1-34.

[21] J.C. Willems. Models for dynamics. Dynamics Reported, 2 (1988), 171-269. MR 90g:58121

[22] S. Zampieri and S.K. Mitter. Linear systems over Noetherian rings in the behavioural approach. Journal of Mathematical Systems, Estimation, and Control, 6 (1996), 235-238.

[23] D. Zelinsky. Linearly compact modules and rings. Amer. J. Math., 75 (1953), 79-90. MR 14:532a

Scuola Normale Superiore, Piazza dei Cavalieri, 7, 56126 Pisa, Italy

E-mail address: fagnani@cibs.sns.it

Dipartimento di Elettronica ed Informatica, Università di Padova, via Gradenigo, 6/A, 35131 PAdova, Italy

E-mail address: zampi@paola.dei.unipd.it 